\title{
DISCLAIMER
}

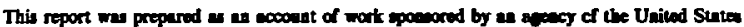

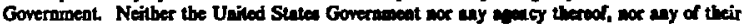
LBL -18690

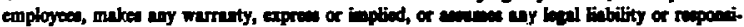

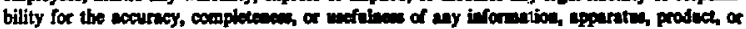

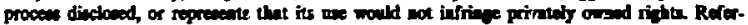

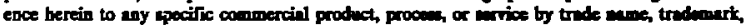

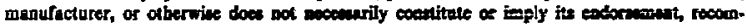
mendation, of farating by the Unitad States Governeat or any arecty theroof. The view

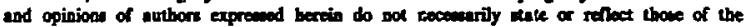
Uniled States Government or any areacy thereor.

\section{AXION MASS LIMITS FROM PULSAR $X$ RAYS}

Donald E. Morris

Lawrence Berkeley Laboratory

University of California

Berkeley, California 94720

December 1984

$\therefore$ 施

"This work was prepared for the US Department of Energy under Contract Number DE-AC: 03-76SF00098 


\title{
Axion Mass Limit fr:at Pulsar X rays
}

\section{Donald Morris}

\begin{abstract}
Axions thermally emitted by a neutron star would be converted into $X$ rays in the strong magnetic field surrounding the star. An improvement in the observational upper limit of pulsed $X$ rays from the Vela pulsar IPSR $0833-45)$ by a factor of 12 would constrain the axion $\pi 1$ ass $\mathrm{M}_{\mathrm{a}}<2 \times 10^{-3} \mathrm{eV}$ if the core is non-superfluid and at temperature $T_{c} \geqslant 2 \times 10^{8} \mathrm{~K}$. If the core is superfluid throughout, an improvement factor of 240 would be needed to provide the same constraint on the axion mass, while in the absence of superfluidity, an improvement factor of 200 could constrain ${ }_{a}<6 \times 10^{-4} \mathrm{eV}$. A search for modulated hard $X$ rays from PSR 1509-58 or other voung pulsars at presently attainable sensitivities may enable the setting of an upper limit for the axion mass. Observation of hard $\mathrm{X}$ rays from a very young hot pulsar with $T_{c} \geqslant 7 \times 10^{8} \mathrm{~K}$ could set a firm bound on the axion mass, since ntuiton superfluidity is not expected above this temperature. The remaining axion mass range $6 \times 10^{-4} \mathrm{eV}>\mathrm{M}_{\mathrm{a}}>10^{-5} \mathrm{eV}$ (the cosmological lower bound) can be covered by an improved Sikivie type laboratory cavitv detector for relic axions constituting the galactic halo.
\end{abstract}




\section{Contents}

1. Introduction

2. Axion to $X$ Ray Conversion by Neutron Stars

3. A. Axion Emission from Neutron Stars

B. Axion Emission from Neutron Stars with Superfluid Cores

C. Axion Mass Limit from Neutron Star Cooling

D. Temperatures of Neutron Stars

4. Efficiency of Conversion into $X$ rays

5. Comparison with Observations
a. The Vela Pulsar
b. The Crab Pulsar
c. PSR 1509-58
d. RCW 103
e. Possible Young Pulsars (age $-10^{2}$ yrs)

6. Conclusions

7. A cknowledgements

References

Table I

Table II

Figure 1 


\section{Introduction}

A natural explanation of the observed CP conservation in strong interactions was, given by Peccei and Quinn', which resulted in the introduction of the axion ${ }^{2}$, a light pseudo-scalar boson. The scheme was extended to grand unification scale $\mathrm{e}^{3}$ and led to the 'invisible' axion with $M_{a} \sim 10^{16}(\mathrm{eV})^{2} / F<I \mathrm{eV}$, where $F$ is the energy scale at which the postulated $P-O$ symmetry is spontaneously broken. Detection of the axion and determination of its mass is important for cosmology as well as elementary particle physics. The axion mass $M_{a}$ has been limited by laboratory experiments and by astrophysical arguments based on the consequences of axion emission on evolution of the $\operatorname{sun}^{5,6}$ to $\mathrm{M}_{\mathrm{a}}<6 \times 10^{-1} \mathrm{eV}$ and of red giant stars ${ }^{5}$ to $\mathrm{M}_{\mathrm{a}}<10^{-1} \mathrm{eV}$. A cosmological lower bound to $\mathrm{M}_{\mathrm{a}} \geqslant 10^{-5} \mathrm{eV}$ arises from the cuntribution of relic axions to the energy density of the universe. ${ }^{7}$ Axions with mass near this lower limit would provide a large fraction of the energy density of the universe in the form of cold dark matter ${ }^{8}$, which could provide the closure density, explain the formation of galaxies and provide the material of galactic halos 9 , and explain 10 the observed large scale structure in the distribution of visible maiter.

Sikivie ${ }^{11}$ has pointed out the electromagnetic interaction of axions and suggested laboratory detectors for solar axions of mass near $10^{-1} \mathrm{eV}$ and for galactic halo axions of mass near $10^{-5} \mathrm{eV}$. The author has proposed an improved detector ${ }^{45}$ for the range $10^{-5} \mathrm{eV}<\mathrm{M}_{\mathrm{a}}<4 \times 10^{-4} \mathrm{eV}$. Moody and Wilczek ${ }^{12}$ have proposed measurement of the extremely weak long range axionic force. ${ }^{5}$ Recently Iwamoto ${ }^{13}$ has considered axion emission from neutron stars. He compared the axion luminosity with neutrino and surface thermal photon luminosities in the standard cooling scheme (no pion condensate or quark matter). He found that the axion luminosity will dominate and shorten the time for cooling to $\mathrm{T} \sim \mathrm{T}_{1}=2 \times 10^{8} \mathrm{~K}$ if $\mathrm{M}_{\mathrm{a}}>1.1-1.7 \times 10^{-\mathrm{Z}} \mathrm{eV}$. This limit is independent of the existence or absence of nuclear superfluidity as explained later. 
Axion to $X$ ray conversion by neutron stars:

In this paper we point out that axions emitted by a neutron star will be converted into $X$ ray photons in the magnetosphere of of the star (see inset of Figure 1). The enormous magnetic field and the interaction distance of several kilometers can result in efficient conversion of axions into photons, despite the extremely weak coupling of the 'invisible' axion. Observational limits on the modulated flux of $X$ rays from pulsars can provide an upper bound on the axion mass. The bound depends on the temperature and possible superfluidity of the neutron star. The axions will have a thermal distribution ${ }^{13}$ at $I_{c}$ the temperature of the core of the star, and convert into $X$ rays with the same energy. (The axions will be red shifted as they travel out to the surface and the $X$ ray photons will be redshifted as they leave the star. ${ }^{14,15}$ )

From the astrophysical limits given earlier, $M_{a}<l e V<<k T$, so the emitted axions will be bighly relativistic. The very small difference between the momentum of the axion and that of the photon is provided by the spatial variation of the mannetic field of the star. Conversion may be quite efficient in the star's extremely strong magnetic field (B $>10^{12}$ gauss) if the field has significant Fourier components with spatial frequencies in the range required for momentum conservation. An axion traveling radially outward through the magnetosphere converts into an $X$ ray traveling in the same direction, since the momentum available from the magnetic field is insignificant compared to the axion momentum. The axion to $X$ ray conversion depends on the transverse component of the magnetic field, (the coupling is proportional ${ }^{11}$ to $E_{x} \cdot B_{*}$ where $E_{X}$ is the $E$ field of the $X$ ray photon, and $B_{*}$ is the $B$ field of the star). The conversion $X$ rays will be polarized and conversion will be most efficient at the magnetic equator of the neutron star where the field is entirely transverse. The $X$ rays reaching the solar system will be modulated as the neutron star rotates. If radio or optical pulsed emission comes from the polar regions, the modulation will be of opposite phase. 


\section{Axion emission from neutron stars:}

First we will calculate the axion luminosity. The result, which is model dependent, will be used in our calculations relating the axion mass to $X$ ray luminosity. Iwamoto ${ }^{13}$ found that the most important process for axion emission from the isothermal core is axion bremsstrablung from neutron-neutron collisions: $n+n \rightarrow n+n+a$, with energy loss rate $\epsilon_{A N N} \propto M_{a}^{2} T^{6} \quad$ (1), from his equation 5 (correcting his expression for $M_{a}$ ). In case of nucleon superfluidity 16,17 this process is strongly suppressed as a result of the energy gap below the critical temperature $\mathrm{T} \sim 7 \times 10^{8} \mathrm{~K}$ where ${ }^{3} \mathrm{P}_{2}$ neutron superfluidity sets in at core densities between 2 and $8 \times 10^{14} \mathrm{~g} / \mathrm{cm}^{3}$. 16 The second important process was found to be axion bremsstrahlung by electrons in the crust: $\mathbf{e}^{-}+$ $(Z, A) \rightarrow e^{-}+(Z, A)+a$, with energy loss rate $\epsilon_{A e e} \propto M_{a}{ }^{2} T^{4} \quad$ (2), from equation 8 of $R \in f, 13$. This process will dominate for $T<2-3 \times 10^{7} \mathrm{~K}$ because of the weaker temperature dependence, and will also dominate at higher temperatures if the first process is suppressed by neutron superfluidity.

I wamoto compared the axion luminosity $L_{a}$ with the neutrino luminosity $L_{v}$ and with the luminosity from surface thermal photon emission $L_{e}$ under various conditions, but did not give numerical estimates for $L_{a^{*}}$ He considered two equations of state: a medjum soft equation of state by Bethe and Johnson $(B J)^{18}$, and a stiff equation of state by Pandharipande and Smith (PS) ${ }^{19}$. The neutrino luminosity had previously been calculated by Soyeur and Brown ${ }^{20}$ using these two equations of state. $r$ alculations for $L_{\nu}$ from the modified URCA process in the core 20,21 give $L_{\nu} \sim 10^{34} \mathrm{erg} / \mathrm{sec}\left(T / T_{1}\right)^{8}$. Iwamoto found that in the absence of nucleon superfluidity, axion emission will dominate the energy loss from neutron stars at $T_{c} \sim T_{1}=2 \times 10^{8} \mathrm{~K}$, if $\mathrm{M}_{\mathrm{a}}>3 \times 10^{-3} \mathrm{eV}$, so from (1), we have

$$
\mathrm{L}_{\mathrm{a}} \sim 1.1 \times 10^{33} \mathrm{erg} / \mathrm{sec}\left(\mathrm{M}_{\mathrm{a}} / 10^{-3} \mathrm{eV}\right)^{2}\left(\mathrm{~T} / \mathrm{T}_{1}\right)^{6}
$$


Axion emission from a neutron star with superfluid core.

lwamoto found that if the neutrons and protons are superfluid throughout the core, the second process (axion luminusity due to bramsstrahlung by electrons in the crust) will dominate, so that at $\mathrm{T} \sim 3 \times 10^{8} \mathrm{~K}$ (BJ) or $2 \times 10^{8} \mathrm{~K}$ (PS) the axion luminosity $\mathrm{L}_{\mathrm{a}}$ will be equal to $\mathrm{L}_{e}$ the photon luminosity for thermal emission from the surface, if $F=$ $6 \times 10^{8} \mathrm{GeV}(\mathrm{BJ})$ or $9 \times 10^{8} \mathrm{GeV}(\mathrm{PS})$, that is if $\mathrm{M}_{\mathrm{a}} \sim 1.7 \times 10^{-2} \mathrm{eV}$ (BJ) or $1.1 \times 10^{-2} \mathrm{eV}(\mathrm{PS})$. Iwamoto used the photon luminosity calculated by Soyeur and Brown 20 , who used the black body formula $L_{e}=4 \pi \delta R_{*}{ }^{2} T_{s}{ }^{4}$, with $R_{*}=11 \mathrm{~km}$ (BJ) or $16 \mathrm{~km}$ (PS) from their table $I$, and with effective surface temperatire $T_{s}\left(\sim T_{c} j^{125}\right)$ taken from figure 2 of Tsuruta $^{22}$. The numerical values are $\mathrm{L}_{\mathrm{e}} \cdots 3 \times 10^{34} \mathrm{erg} / \mathrm{sec}$ at $\mathrm{T}=3 \times 10^{8} \mathrm{~K}(\mathrm{BJ})$ or $1 \times 10^{34} \mathrm{erg} / \mathrm{sec}$ at $\mathrm{T}=2 \times 10^{8} \mathrm{~K}$ (PS). Then from reletion (2),

$$
\begin{aligned}
& \mathrm{L}_{\mathrm{a}} \sim 2 \times 10^{31} \mathrm{erg} / \mathrm{sec}\left(\mathrm{M}_{\mathrm{a}} / 10^{-3} \mathrm{eV}\right)^{2}\left(\mathrm{~T} / \mathrm{T}_{1}\right)^{4} \quad \text { (4) Eor (BJ), or } \\
& \sim 10 \times 10^{32} \mathrm{erg} / \mathrm{sec}\left(\mathrm{M}_{\mathrm{a}} / 10^{-3} \mathrm{eV}\right)^{2}\left(\mathrm{~T} / \mathrm{T}_{1}\right)^{4} \quad \text { (5) for (PS), which has a thicker crust. }
\end{aligned}
$$

\section{Axion Mass Limits from Neutron Star Cooling}

Although axion emission will dominate ${ }^{13}$ the energy loss near $\mathrm{T}_{1}$ if $\mathrm{M}_{\mathrm{a}}>3 \times 10^{-3}$ in the absence of nucleon superfluidity in the core, inspection of the cooline curves calculated by several authors ${ }^{23-26}$ for a range of nonsuperfluid models (see Table I) indicates that a moderate increase in cooling rate would lead to a temperature at a given age which is still within the range of uncertainty of the cooling calculations. So observations of the surface temperature would not confirm or contradict axion emission. For example, let $M_{a}=10^{-2} \mathrm{eV}$, then from (3), at $T=7 \times 10^{8} \mathrm{~K}, \mathrm{~L}_{\mathrm{a}} \sim \mathrm{L}_{\nu}=$ $2 \times 10^{38} \mathrm{erg} / \mathrm{sec}$, and at $\mathrm{T}=\mathrm{T}_{1}=2 \times 10^{8} \mathrm{~K}, \mathrm{~L}_{\mathrm{a}} \sim 10^{35} \mathrm{erg} / \mathrm{sec} \sim 11 \mathrm{~L}$. Consider model $\mathrm{V}_{\gamma} \mathrm{II}$ (nonsuperfluid) of Ref 23, temperatures for this model are given in Table 1 . The calculated jncrease of the cooling rate caused by axion emission below $\mathrm{T}=7 \times 10^{8} \mathrm{~K}$ would lead to $\mathrm{T} \sim \mathrm{T}_{1}$ at $\mathrm{t} \sim 10^{4}$ years, instead of at $\mathrm{t} \sim 10^{5}$ years, giving a corresponding $\mathrm{T} e$ 
$\sim 1.6 \times 10^{\circ} \mathrm{K}$, which is quite consistant with observation. Similarly, in the superfluid case, although axion emission would dominate ${ }^{13}$ for $10^{9} \mathrm{~K}>\mathrm{T}>3 \times 10^{8} \mathrm{~K}$ (BJ) or $4 \times 10^{9}>\mathrm{T}>$ $2 \times 10^{8} \mathrm{~K}$ (PS) the total luminosity would increase by a factor less than ten for Mas $10^{-2} \mathrm{eV}$. This would still permit surface temperatures compatible with observation at ages of $10^{3}$ and $10^{4}$ years. We conclude that the limit on axion mass placed by cooling of neutron stars is $M_{a}<1-2 \times 10^{-2} \mathrm{eV}$ for both superfluid and non superfluid models.

\section{Temperature of neutron stars}

The emission of axions is strongly dependent on temperature, but cooling calculations for different neutron star models with various equations of state, with superfluid and nonsuperfluid cores, and with various magnetic fields all give core temperatures between 1.5 and $4 \times 10^{8} \mathrm{~K}$ at age $t \sim 10^{4}$ years (see Table 1 ). The dependence of thermal conductivity of the crust on magnetic field is very weak, and does not affect the core temperature significantly. ${ }^{27}$ It is interesting to note that the predicted surface temperature as seen by a distant observer $\mathrm{T}_{\mathrm{e}}^{\infty} \sim 1.2-3 \times 10^{6} \mathrm{~K}$ at age $10^{4}$ years, quite consistant with the value $\mathrm{T}_{\mathrm{e}}^{\infty} \sim 1.5 \times 10^{6} \mathrm{~K}$ from observation of non pulsed sof $\mathrm{x}$ ray luminnsity from the Vela pulsar, 28 although the emission has not yet been shown to have a thermal distribution. Similarly, for age $t \sim 10^{3} \mathrm{yrs}$, the predicted $\mathrm{T}_{\mathrm{e}}^{\infty} \sim 1.8-6 \times 10^{6} \mathrm{~K}$, is comparable to $\mathrm{T}_{\mathrm{e}}^{\infty} \sim 2 \times 10^{6} \mathrm{~K}$ from observation of the Crab pulsar. ${ }^{29}$ Alternatively, we may estimate the core temperature $T_{c}$ from observations of $T_{e}^{\infty}$. Following the most recent analysis ${ }^{14}$ relating $T_{c}$ and $T_{e}^{\infty}$ we expect $T_{c} \sim 2.8-3.6 \times 10^{8} \mathrm{~K}$ for an observed $\mathrm{T}_{\mathrm{e}}^{\infty} \sim 1.5 \times 10^{6} \mathrm{~K}$ (Vela pulsar) and $\mathrm{T}_{\mathrm{C}} \sim 4.5-6 \times 10^{8} \mathrm{~K}$ for an observed $\mathrm{T}_{\mathrm{e}}^{\infty} \sim 2 \times 10^{6} \mathrm{~K}$ (C.rab pulsar). (A much lower estimate of $T_{C} \sim 0.5-2.5 \times 10^{7} \mathrm{~K}$ for the Vela puisar has been made ${ }^{30}$ from analysis of post glitch relaxation in terms of vortex creep in the superfluid core.) 
Efficiency of conversion into $X$ Rays:

A general expression for the axion to $x$ ray conversion cross section is given in equation 6 of ref 11

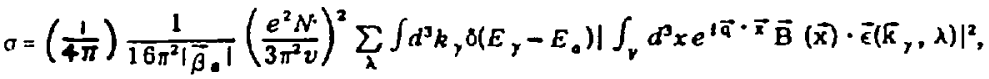

where $q=k_{x}-k_{a}=\left(M_{a} c^{2}\right)^{2} / 2 \hbar c E_{a} \quad$ (7), as required for momentum conservation since the axions are highly relativistic. ${ }^{31} \quad E_{a} \sim 1.8 \mathrm{kT} \mathrm{C}_{c}$ at the peak of the Plankian distribution. The factor $(1 / 4 \pi)$ is a correction given by Sikivie. A simple method to calculate the cross section $\sigma$ is to use the results of Ref 11 for solar axion conversion in a laboratory detector. We divide the number of $x$ rays/sec produced in a laboratory detector by conversion of solar axions: $(1 / 4 \pi) 6 \times 10^{-3} \mathrm{~S} \mathrm{~L}^{2}\left(10^{8} \mathrm{GeV} / \mathrm{u}, 4(\mathrm{~B} / 10 \mathrm{~T})^{2} \mathrm{~N}^{2}(8 \pi\right.$ $R / E_{a} L$ )/sec (equation 13 of Ref 11 ), by the solar axion flux: $0.8 \times 10^{17}\left(10^{8} \mathrm{GeV} / \mathrm{v}\right)^{2} / \mathrm{m}^{2}$ sec (equation 3 of Ref 11). We find

$$
\sigma=1.4 \times 10^{-23} \mathrm{SL}^{2}\left(\mathrm{M}_{\mathrm{a}^{\prime}} / 10^{-3} \mathrm{eV}\right)^{2}(\mathrm{~B} / 10 \mathrm{~T})^{2} \mathrm{R}^{\prime}
$$

where $L$ is the length of the detector in meters, $S$ is the area in sq. meters, and the result is expressed in terms of $M_{a}$ by equation 2 of $R$ ef $11: M_{3}=1.24 \times 10^{-3} \mathrm{eV}\left(10^{10} \mathrm{rieV}_{\mathrm{v}}\right)(\mathrm{N} / 6)$. The response function $\mathrm{R}^{\prime}$ of the detector is equal to the square of the Fourier transform of the normalized magnetic field inside the detection region. (In terms of $\mathrm{R}$ of equation 12 of Ref. 11, $R^{\prime}=8 R / E_{a}{ }^{L .}$ ) To evaluate $\sigma$ at the neutron star, we must substitute the square of the Fourier transform of the normalized magnetic field in the magnetosphere outside of the star for the expression given in Ref. 11. We find the required Fourier transform below. 
The field distribution may be approximated by a dipole field:

$$
B(r)=\left(B_{d} / 2\right)\left(R_{*} / r\right)^{3}\left(\hat{\theta}_{\sin \theta}+2 \hat{r} \cos \theta\right) \text { for } r>R_{*},
$$

where $r$ is the radial distance from the center, $R_{*}$ is the radius of the star, $\theta$ is the polar angle from the magnetic field axis, and $B_{0}$ is the (radial) field at the pole of the star. We note that the magnetic field at the equator $B_{R}=B_{0} / 2$. For an oblique rotator with magnetic axis at angle $\alpha$ to the rotation axis, $B_{0}$ may be determined ${ }^{32}$ from the pulsar braking: $B_{0}=\left(3 I c^{3} P \dot{P} / 8 \pi^{2}\right)^{1 / 2} R_{*}{ }^{3} \sin ^{-1} \propto$. We will take $I \sim 10^{45}\left(R_{*} / 10 \mathrm{Km}\right)^{2} \mathrm{gm} \mathrm{cm}^{2}$.

Since an axion will convert into an $X$ ray photon traveling in the same direction, we will treat the magnetic fiel $t$ and its transform in one dimension. Axions are emitted throughout the core (in the crust in case of superfluidity), so we should integrate over all parallel axion-photon paths through the magnetosphere which originate inside the core (crust) of the star. The spatial variation of the transverse magnetic field along such paths will be similar, yielding similar spatial frequency distributions. For simplicity we will consider only a central path passing radially through the magnetic equator, where $B(r)=B_{R} b(r) \hat{\Theta}=\left(B_{0} / 2\right) b(r) \hat{\theta}$, with $b(r)=\left(R_{\star} / r\right)^{3}$.

The Fourier transf orm of $b(r)$ over the range $R_{*}<r<\infty$ has been approximated by a discrete Fourier transform $\underline{b}(O)$, where $O=R * q$, with power distribution $\underline{b}^{2}(C)$ shown in figure 1. (We note that to a good approximation $b^{2}(O) \sim\left(2.3+0^{1.2},-5 / 3\right.$.) The integral of $B(r)$ over $R_{*}<r<\infty$ is equal to $R_{*} B_{R} / 2$, while in the limit $O \rightarrow 0, \underline{b}^{2}(O) \rightarrow 0.5$ so that $\underline{E}^{2}(Q) \rightarrow 0.25$ (see figure 2 ), therefore we set $L=R_{*}$ in equation 13 of $R$ ef 11 to be consistant with the normalization of $\underline{b}^{2}(O)$. The magnetic field of the neutron star may contain higher multipole components ${ }^{33}$, which do not contribute to the braking since they fall off rapidly with distance from the star. This would increase $\underline{b}^{2}(O)$ for $\rho>2 \pi$, so we can consider the curves in figure 1 as giving lower limits at large $O$. The possible 
existence of very large magnetic fields inside the star $^{34}$ would not cause the conversion of axions because the very short photon mean free path $\left(<<R_{*}\right)$ inside the star would suppress the coherent conversion of axions into photons.

Substituting in (8) and dividing by the area $S$, we find the fraction $\sigma / S$ of the incident axions which will convert into $X$ rays in the magnetosphere of the star:

$$
\sigma / \mathrm{S} \sim 0.035 b^{2}(Q)\left(M_{a^{\prime}} 10^{-3} \mathrm{eV}\right)^{2}\left(B_{d} / 10^{12} G_{a u s s}\right)^{2}\left(R_{*} / 10 \mathrm{Km}\right)^{2}
$$

The dependence of $\sigma / S$ on $O$ is indicated by curve $Q \underline{b}^{2}(O)$ in Figure 1 , since $\sigma / S \propto$ $b^{2}(0) M_{a}{ }^{2}$ from $(10)$ and $M_{a}{ }^{2} \propto q \propto O$ from (7). However, the conversion efficiency cannot exceed 0.5 due to reconversion of $X$ says into axions, which was not considered in (6). The correct expression for the conversion efficiency $\eta=(1 / 2)\left(1-\mathrm{e}^{-2 \nabla / 5}\right.$, (11). We also note that when $\sigma / S>0.5$ in (10), the modulation of the $x$ ray signal with rotation of the pulsar will be suppressed. From $(10)$ and Figure 1 , for $R_{*} \sim 10 \mathrm{Km}$, this can only happen if $\mathrm{B}_{\mathrm{o}}>7 \times 10^{12}$ gauss, and will occur near $M_{\mathrm{a}} \sim 1-2 \times 10^{-3} \mathrm{eV}$.

For sufficiently small $B_{0}$ (giving $\sigma / S<0.5$ ), the axion luminosity $L_{a} \propto M_{a}^{2} \propto 0$ from (3), (4), (5), and (7), so the $X$ ray luminosity from axion conversion $L_{x}=\eta L_{3} \propto$ $\mathrm{O}^{2} \underline{\mathrm{b}}^{2}(O)$, which is plotted in figure 1 . This function indicates the dependence of the expected $X$ ray luminosity on axion mass. It is nearly constant above $O=10$ (where $M_{a} \sim$ $3 \times 10^{-3} \mathrm{eV}$ if $\operatorname{T\sim T}_{1}=2 \times 10^{8} \mathrm{~K}$ ), but decreases rapidly for smaller $O$ (and $M_{a}$ ).

For $M_{a}<7 \times 10^{-4} \mathrm{eV}, 0<0.4$ and from figure $1, b^{2}(O) \rightarrow 0.25$. Then, so long as $\sigma / \mathrm{s}<$ 0.5 in (10), i.e. for $B_{0}<7 \times 10^{12}\left(10^{-3} \mathrm{eV} / \mathrm{M}_{\mathrm{a}}\right)$ gauss, and the core is not superfluid, we may combine (10) and (3) to give

$$
\mathrm{L}_{x} \sim 1 \times 10^{31} \mathrm{erg} / \mathrm{sec}\left(\mathrm{M}_{\mathrm{a}} / 10^{-3} \mathrm{eV}\right)^{4}\left(\mathrm{~B}_{\mathrm{o}} / 10^{12} \text { gauss }\right)^{2}\left(\mathrm{R}_{*} / 10 \mathrm{Km}\right)^{2}\left(\mathrm{~T} / \mathrm{T}_{1}\right)^{6}
$$


Comparison with observations.

We shall attempt to use observational limits on the modulated $X$ ray flux from pulsars in conjunction with the preceeding analysis to place upper limits on $\mathrm{M}_{\mathrm{a}^{*} \text {. Our }}$ conclusions will be model dependent becasse axion emission is suppressed when thc neutrons are superfluid throughout the core.

The Vela pulsar (PSR0833-45): The best limit to modulated $X$ ray arnission of a reasonably young neutron star is for the Vela pulsar(PSR 0833-45) by Knight et al. ${ }^{35}$ who found that the pulsed flux is less than $5 \times 10^{-6} / \mathrm{Kev} \mathrm{cm}^{2}$ sec (3o) over the range 15-175 $k e V$, i.e. $<5 \times 10^{-4}$ photons $/ \mathrm{cm}^{2}$ sec (95\% confidence level). For $T=T_{j}$ and a redshift

$\sim 1.16$, the energy of observable redshifted $X$ ray photons from axion canversion would be $\sim 1.8 \mathrm{kT} T_{1} \times 0.86 \sim 2 ; \mathrm{keV}$. With this average photon energy, and since the distance of the Vela pulsar is $\sim 500$ parstec, the corresponding $(95 \%(\mathrm{~L})$ upper limit on modulated $X$ rav luminosit $y^{n}$ is $\mathrm{L}_{x}<7 \times 10^{32}$ erg/sec.

This may be compared with the calculated $X$ ray lumirosity from axion convarsion given in Table I. The calculations are for models (BJ) and (PS) with $M_{*}=1.3 \mathrm{M}_{\mathrm{O}}$ (solar mass), and with $\mathrm{T}=\mathrm{T}_{1}=2 \times 10^{8} \mathrm{~K}$. For the nonsuperfluid models we see that to constrain tha axion mass to $M_{a}<2 \times 10^{-3} \mathrm{eV}$, improvement in the pres nt observational limit by a factor of 5 (BJ) or 12 (FS) is needed. To constrain $M_{\mathrm{a}}<6 \times 10^{-4} \mathrm{eV}$ will require an improvement by a factor of 100-200 (examples 5 \&6). This sensitivity may be approached by the zext generation of $X$ ray telescopes. 36

If the neutrons and protons are superfluid throughout the core axion emission is considerably reduced, so that to set a limit of $M_{a}<2 \times 10^{-3} \mathrm{eV}$ lexamples 3 and 4 of Table II), we require improvement of the present observational limit by a factor of $140-230$. Examples 7 and 8 give $L_{x}^{\infty}$ calculated for $M_{a}=1 \times 10^{-3} \mathrm{eV}$ in superfluid models, the required factor of improvement would be 500-1100. 
The core neutrons may be nonsuperfluid in part of the star ${ }^{16}$ between the density ranges for ${ }^{1} S_{0}$ superfluidity $\left(1 \times 10^{11}-1.5 \times 10^{14} \mathrm{~g} / \mathrm{cm}^{3}\right)$ and ${ }^{3} P_{2}$ superfluidity $\left(2 \times 10^{14}\right.$. $8 \times 10^{14} \mathrm{~g} / \mathrm{cm}^{3}$ ). The core neutrons may be nonsuperfluid at densities above $8 \times 10^{14} \mathrm{~g} / \mathrm{cm}^{3}$ which are reached in models with softer equations of atate kuch as BJ. ${ }^{36}$ Axion emission would take place by process (1) from the nonsuperfluid region, leading to a limit on the axion mass similar to the nonsuperfluid case without requiring $\mathrm{s}$ irch a large improvement in the $X$ ray observations.

The Crab Pulsar(PGR0531+21): The pulsed $X$ ray emission over the range $45-110 \mathrm{KeV}$ is $2.1 \pm 0.7 \times 10^{-4}$ photons $/ \mathrm{KeV} \mathrm{\textrm {cm } ^ { 2 }}$ sec. 37 If $\mathrm{T}_{\mathrm{c}} \sim 4 \times 10^{8} \mathrm{~K}, \mathrm{E}_{\mathrm{a}} \sim 64 \mathrm{KeV}$, then the energy flux $\sim 1.4 \times 10^{-9} \mathrm{erg} / \mathrm{cm}^{2}$ sec. The distance $\mathrm{d} \sim 2 \times 20^{3}$ parsecs, so the luminosity is $7 \times 10^{35} \mathrm{erg} / \mathrm{sec}$. From figure $2 \mathrm{~b}$ of Ref 37 the sinusoidal part of the pulsed flux $640 \%$, so

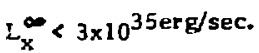

The predicted core temperature from Table I is $T_{c} \sim 3.3-8 \times 10^{8} \mathrm{~K}$. We take $\mathrm{T}_{c}=4 \times 10^{8} \mathrm{~K}$ and neglect superfluidity. Then we find $\mathrm{L}_{\mathrm{a}} \sim 2.8 \times 10^{35} \mathrm{erg} / \mathrm{sec}$ for $\mathrm{M}_{\mathrm{a}}=$ $2 \times 10^{-3} \mathrm{eV}$ from (3) and the calculated $\mathrm{l}_{\mathrm{x}}^{\infty}$ from axion conversion ${ }^{38}$ is $3(1.2) \times 10^{34} \mathrm{erg} / \mathrm{s}$ for BJ (PS). Even for a larger axion mass the calculated $X$ ray flux from axion conversion is smaller than the measured pulsed $X$ ray flux, so observation of the Crab pulsar cannot set any axion mass limit.

PSR 1509-58: This pulsar has a spin down age similar to the Crab, and a distance of about $4.2 \times 10^{3}$ parsecs. It was recently discovered as a soft $X$ ray $^{39}$ and radio ${ }^{40}$ pulsar. The soft $X$ ray data has been summarized and evaluated. 41 The increase of calculated axion emission at the (presumed) higher temperature of this younger neutron star can offset the reduction in $X$ ray flux at the solar system due to the larger distance. This pulsar has a large $\dot{P}$ which gives $B_{0} \sim 12 \times 10^{12}$ gauss for model BJ or $B_{0} \sim 6 \times 10^{12}$ gauss for model PS. The axion to $X$ ray conversion is limited since $\eta$ saturates at 0.5 , and as pointed out earlier, the modulation of the $X$ ray fux will be suppressed. We roughly 
estimate the degree of suppression by comparing the conpersion effeciency $\eta$ in the equatorial field $B_{R}=B_{\alpha} / 2$ when the magnetic equator is in line with the observer, with the efficiency for a transverse field of half this value $\eta_{1 / 2}$, to model the reduction of the transwerse field component as the pulsar rotates. We take $T_{c} \sim 4 \times 10^{8} \mathrm{~K}$ as for the Crab pulsar, so for $M_{a}=2 \times 10^{-3} \mathrm{eV}, L_{a} \sim 2.8 \times 10^{35} \mathrm{erg} / \mathrm{sec}$ and $O=1.75(2.6)$ for $\mathrm{BJ}$ (PS) as for the Crab. Fros (10), $\sigma / S \sim 2.07(0.77)$ for BJ (PS). The conversion effeciency $\boldsymbol{q}=0.49$ (0.39) while $\eta_{1 / 2}=0.32(0.16)$. We estimate the $X$ ray modulation to be $\eta-\eta_{1 / 2}=0.17$ for $\mathrm{BJ}$ (or 0.23 for PS). The resulting $X$ ray flux at the solar system is $1.8(2.8) \times 10^{-4}$ icm ${ }^{2} \sec$ for $B J$ (or PS). We see that if the modulated hard $X$ ray flux from this pulsar is $<1.8 \times 10^{-4} / \mathrm{cm}^{2} \mathrm{sec}$ then $M_{a}<2 \times 10^{-3} \mathrm{eV}$ if the pulsar cole is not superfluid. This requires a threefold improvement in meesurement sensitivity compared with the existing Vela observations. In superfluid models $L_{a} \sim 1.25(6.4) \times 10^{33} \mathrm{erg} / \mathrm{sec}$ for $\mathrm{BJ}$ (PS) from (4) and (5), giving a flux of $8(64) \times 10^{33} / \mathrm{cm}^{2}$ sec. This requires a $600-\mathrm{fold}$ (80-fold) increase in measurement sensitivity to limit $M_{a}<2 \times 10^{-3} \mathrm{eV}$. No search for pulsed bard $X$ rays from this pulsar have yet been made to the authors knouledge. A good upper limit on modulated $X$ ray luminosity from this pulsar could set an axion mass limit. 49

RCW 103: This $X$ ray source may be a hot neutron star at $d \sim 3.3 \times 10^{3}$ parsecs, since sof $t$ $X$ ray observations ${ }^{42}$ are consistant with $T_{e}^{\infty} \sim 2 \times 10^{6} \mathrm{~K}$.

Young pulsars: The rate of pulsar formation in the galaxy ${ }^{43}$ may be $1 / 20$ to $1 / 80$ per year. Young pulsars would have hot cores, and the hard ( $1100 \mathrm{keV}) \mathrm{X}$ rays from axion conversion would penetrate the galaxy. At age $t \sim 10^{2} y$ the expected core temperature $T_{c} \sim 6-10 \times 10^{8} \mathrm{~K}$ (Table I). This may be sufficient to extinguish any ${ }^{3} P_{2}$ superfluidity of the core neutrons and eliminate suppression of axion emission caused by superfluidity. For $\mathrm{T}_{\mathrm{c}} \sim 7 \times 10^{8} \mathrm{~K}$ and $\mathrm{M}_{\mathrm{a}} \sim 2 \times 10^{-3} \mathrm{eV}, \mathrm{L}_{\mathrm{a}} \sim 8 \times 10^{36} \mathrm{erg} / \mathrm{sec}$ and $\mathrm{E}_{\mathrm{a}} \sim 110 \mathrm{KeV}$. We assume that the pulsar magnetic field (which can be determined from P户े) has reached at least $3 \times 10^{12}$ gauss within that time. Then for model BJ (PS), $Q \sim 1.0(0.5), \eta-q 1 / 2=0.12(0.19)$ 
and $\mathrm{L}_{\mathrm{X}}^{\infty} \sim 7(12) \times 10^{35} \mathrm{erg} / \mathrm{sec}$. For a typical distance $\mathrm{d} / \mathrm{N} 1.3 \times 10^{4}$ parsecs we find the modulated flux at the solar system measured over a $10^{2}$ keV range would be $\sim 2.2(4) \times 10^{-6}$ photons/keV $\mathrm{cm}^{2} \mathrm{sec}$, (the same order as the present detection limit for pulsed $X$ rays from the Vela pulsar).

A young pulsar, if found, could be very useful in setting an axion mass limit. There exist strong galactic $X$ ray sources ${ }^{44}$ of uncertain nature with flux of about $5 \times 10^{-5} / \mathrm{Kev} \mathrm{cm}^{2}$ sec. A search for periodicity in these sources mould be necessary to convirm that they are pulsars, evaluate their magnetic field strength, and set a limit on modulated $X$ ray flux. Very recently, D. A. Green and S. F. Gull ${ }^{48}$ have reported a 'filled center' supernova remnant G227.1+1.0 with possible pulsar only a few hundred years old, and Jikely distance $d \sim 10^{4}$ parsecs, which may be suitable.

\section{Conclusions.}

Axion emission by neutron stars can be de:ected by observation, unlike neutrino emission, since axions are efficiently converted irto $X$ rays in the magnetosphere of the star. The production of axions in neutron stars and the conversion efficiency both increase with axion mass, so upper limits can be placed on the axion mass $M_{a}$ from observational limits to modulated $X$ ray flux from pulsars.

Improvement in observational limits on modulated $X$ ray emission from the Vela Pulsar appear to offer the best prospects for limiting the axion mass to below $2 \times 10^{-3} \mathrm{eV}$. In the absence of nucleon superfluidity in the core, and assuming standard cooling, which gives a core temperature $T_{c} \sim 2 \times 10^{8} \mathrm{~K}$ for the Vela pulsar (no pion condensation or quark matter), an improvement in the present observational limit on the modulated $X$ ray flux by a factor of 12 can limit the axion mass to less than $2 \times 10^{-3} \mathrm{eV}$ if pulsed $X$ ray emission of non-arion origin from the pulsar magnetosphere does not interfere. Future $X$ rav telescopes with 230 fold increase in sensitivity may establish limits for $\mathrm{M}_{\mathrm{a}}<2 \times 10^{-3} \mathrm{eV}$ 
(for models with superfluid neutrons throughout the core), or for non superiluid models, $M_{a}<6 \times 10^{-4} \mathrm{eV}$. Measurements of modulated $X$ ray emission from PSR 1509-58 or a still younger puisar may provide a limit on the axion mess. A bound from a very young hot pulsar would be independent of possible superfluidity of the core at lower temperatures. The required sensitivity is on the same order as that already achieved in the measurements of the Vela pulsar. The remaining allowed range of axion mass $6 \times 10^{-4} \mathrm{eV}>\mathrm{M}_{\mathrm{a}}>10^{-5} \mathrm{eV}$ (the cosmological lower bound) may he covered by an improved wide range Sikivie type laboratory cavity detector ${ }^{45}$ wich can detect relic axions if they make up the galactic halo.

\section{Acknowledgements.}

The author would iike to thank P. Sikivie, F. Wilczek, J. Mnody, H. Quinn, and S. Burns for stimulating discussions about axions, J. Arons and D. Backer for information about pulsars, and F. Knight, R. Rothschild, F. Marsball, D. Helfand and F. Seward for information about $X$ ray observations of neutron stars. Thanks are due to J. Machol who computed the discreet Fourier transform and the functions in figure 1 . The author would also like to thank $C$. Pennypacker for stimulating his interest in axion hunting, and especially R. A. Muller and L. W. A lvarez for continuing encouragement. 


\section{References}

$1_{\mathrm{K} .}$ D. Peccei and H. Quinn, Phys. Rev. Lett. 38, 1440 (1977), and Phys. Rev.D 16, 1791 (1977).

${ }^{2}$ S. Weinberg, Phys. Rev. Lett. 40, 223 (1987); F. Wilczek, Phys. Rev. Lett. 40, 279 (1978).

3. E. Kim, Phys. Rev. Lett. 43, 103 (1979); M. Dine, W. Fischler, and M. Srednicki, Phys. Lett. 104B, 199 (1981).

${ }^{4}$ F. Reines et al., Phys. Rev. Lett. $\underline{37}, 325$ (1976).

${ }^{5}$ D. A. Dicus, E. W. Kolb, V. L. Teplitz, and R. V. Wagoner, Phys. Rev. D $\underline{18,1829}$ (1978), and 22, 839 (1980); M. Fukugita, S. Watamura, and M. Yoshimura, Phys. Rev. Lett. 48. 1522, (1982), and Phys. Rev. D 26, 1840 (1982); J. Ellis and K. A. Olive, Nucl. Phys. B223, 252 (1983);

${ }^{6}$ L. M. Krauss, J. E. Moody, and F. Wilczek, Phys. Lett. 144B, 391 (1984).

7J. Preskill, M. B. Wise, and F. Wilczek, Phys. Lett. 120B, 127 (1983; L. F. Abbott and P. Sikivie. Phys. Lett. 120B, 133 (1983; M. Dine and W. Fischler, Phys. Lett. 120B. 137 (1983).

${ }^{8}$ For a review, see G. R. Blumenthal, S. M. Faber, J. R. Primacl, and M. J. Rees, N ature $311,517(1984)$

9J. Ipser and P. Sikivie, Phys. Rev. Lett. 50, 925 (1983); F. W. Stecker and O. Shafi, Phys. Rev. Lett. 50, 928 (1983); M. Yoshimura, Phys. F. ev. Lett. 51, 439 (1983); M. Fukugita and M. Yoshimura, Phys. Lett. 127B, 181 (1983).

${ }^{10}$ P. Sikivie, Phys. Rev. Lett. $\underline{48}, 1156$ (1982); G. Lazarides and C. Shafi, Phys. Lett. 115B, 21 (1982).

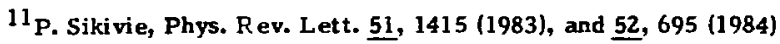

12 J. E. Moody and F. Wilczek, Institute for Theoretical Physics, Santa Barbara, Report No.NSF-ITP-83-177.

${ }^{13}$ N. I wamoto, Phys. Rev. Lett. $\underline{53}, 1198$, (1984). 
14E. H. Gudmundsson, C. J. Pethick, and R. I. Epstein, Ap. J. 272, 286 (1983)

${ }^{15}$ L. Lindblom, Ap. J. 278, 364, (1984).

${ }^{16}$ T. Takatsuka, Prog. Theor. Phys. 48, 1517 (1972)

${ }^{17}$ N.-C. Chao, J. W. Clark and C.-H. Yang, Nucl. Phys. A179, 320 (1972)

${ }^{18}$ H. A. Bethe and M. B. Johnson, Nucl. Phys. A230, 1 (1974)

${ }^{19}$ V. R. Pandharipande and R.A. Smith, Nucl. Phys. A237, 507 (1975)

${ }^{20} \mathrm{M}$. Soyeur and G. E. Brown, Nuclear Physics A324, 464 (1979), figs. 7 and 8 (BJ) and fig. 9 (PS).

${ }^{21}$ B.L. Friman and O.V. Maxwell, Ap. J. 232, 541 (1979), eqs. $67 \mathrm{c}$ and 75; J. N. Bahcall and R. A. Wolf, Phys. Rev. 140B, 1445 (1965), 140B, 1452 (1965). Ap.J. 142, 1254 (1965)

${ }^{22}$ S. Tsuruta in "Physics of dense matter" (AIU Symposium No.53, 1972), (Riedel 1974),

23 ibid. Refer to figures 3 and 4, curves $I$ and II, the other curves are not relevent since the magnetic field dependence of the cooling has subsequently been found to be very weak. 27

${ }^{24}$ S. Tsuruta, Physics Reports $\underline{56}, 237$ (1979)

${ }^{25}$ K. Nomoto and S. Tsuruta,AP. J. 250, L19 (1981)

${ }^{26}$ M. B. Richardson, H. M. Van Horn, K. F. Ratcliff, and R. C. Malone, A.J.J 255, 624 (1982)

${ }^{27}$ L. Hernquist, Ph. D. Thesis, unpublished, Calif ornia Institute of Technology, 1984.

${ }^{28}$ F. R. H arndon, et.al., Bull. Am. Astron. Soc. 11, 789 (1979)

${ }^{29}$ F. R. Harnden, et.al., Bull. A m. A stron. Soc. $\underline{11}, 424$ (1979)

${ }^{30}$ M. A. Alpar, P. W. Anderson, D. Pines, and J. Shaham, Ap. J. 276,325 (1984)

${ }^{31}$ For the $X$ ray photon $E_{x}{ }^{2}=P_{x}{ }^{2} c^{2}=E_{a}{ }^{2}=\left(M a{ }_{a}{ }^{2}\right)^{2}+P_{a}{ }^{2} c^{2}$ for the axion, or $P_{x}=$ $P_{a}\left(1+2\left(M_{a}{ }^{2} c^{2} / 2 P_{a}^{2}\right)\right)^{1 / 2}$ and $M_{a} \ll P_{a} / c$, so $q=\left(P_{x}-P_{a}\right) h=\left(M_{a} c^{2}\right)^{2} / 2$ KcE as piven in equation 11 of Ref 11 .

32 F. Pacini, Nature $\underline{221} 454$ (1968) 
${ }^{33}$ M. A. Ruderman and P. G. Sutherland, Nature Phys. Sci. 246, 93 (1973)

${ }^{34}$ M. A. Ruderman, Ann. Rev. Ast . Astrophys. 10,427 (1972)

${ }^{35}$ F. Knight, J. L. Matteson, L. E. Peterson, and R. E. Rothschild, Ap.J. 260, 553 (1982)

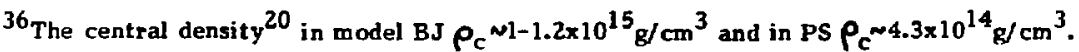
(Pion condensate is unlikely below $4 \times 10^{14} \mathrm{~g} / \mathrm{cm}^{3}$ : O. Maxwell, et.al., Ap. J. 216,77 (1977) ${ }^{37}$ R. B. Wilson and G. J. Fishm an, Ap. J. 269, 273 (1983)

${ }^{38_{F}}$ For model $\mathrm{BJ}, \mathrm{B}_{\mathrm{O}} \sim 3.14 \times 10^{12}$ gauss, $O \sim 1.75$, and $\eta 0.15$, giving a prediction of $\mathrm{L}_{\mathrm{x}}^{\infty}$ $3 \times 10^{34} \mathrm{erg} / \mathrm{sec}$. For model PS, $B_{0} \sim 1.5 \times 10^{12}$ gauss, $Q \sim 2.6$, and $\eta \sim 0.05$, giving a prediction of $L_{x}^{\infty} \sim 1.2 \times 10^{34} \mathrm{erg} / \mathrm{s}$. For higher axion mass, $L_{x}^{\infty} \propto \mathrm{O}^{2} \underline{b}^{2}(O)$ saturates at ? 5-3 times the values calculated for $M_{a}=2 \times 10^{-3} \mathrm{eV}$ (See Figure 1).

${ }^{39}$ F. D. Sew ard and F. R. Harnden, Ap. J. Lett. $\underline{256}$, L45 (1982).

${ }^{40}$ R. N. Manchester, I. R. Tuohy, and N. D'A mico, A p. J. Lett. 262, L.31 (1982).

${ }^{41}$ F. D. Seward, F. R. Harnden, A. Szymkowiak, and J. Swank, Ap. J. 281, 650 (1984)

42I. R. Tuohy and G. P. Garmire, Ap. J. Lett., 239, L107 (1980); I. R. Tuohy, G. P. Garmire, R. N. Manchester, and M. A. Dopita, Astrophys. 268, 778 (1983)

43 H. L. Shipmari and R. F. Green, Ap. J. Lett. 239, L111 (1980); G. A. Tammann, in "Supernovae", p371 (ed. M. J. Rees and R. S. Stoneham), Reidel (1972) 44 A. M. Levine, et. al., Ap. J. Suppl. $\underline{54}$, No 4 (1984), tables 2 and 10). ${ }^{45} \mathrm{D}$. Morris, Lawrence Berkeley Lab. Report LBL-17915 (May 1984) ${ }^{46} \mathrm{G}$. Glen and P. Sutherland, Ap. J. $\underline{239}, 671$ (1980) ${ }^{47}$ K. A. Van Riper and D. G. Lamb, Ap. J. 244, L1 3 (1981) ${ }^{48}$ D. A. Green and S. F. Gull, Nature $\underline{312}, 527$ (1984) 
49 Reduction of the theoretical axion coupling would not reduce the godulated $x$ ray flux proportionately, since $\eta$ is saturated in the high magnetic field. For example, if we reduce the cross section $\sigma$ by a factor of 4 , we find $\sigma / S=0.52(0.19), \eta=0.32(0.16)$,

$\eta_{1 / 2}=0.11(0.046)$, and $\eta-\eta_{1 / 2}=0.21\{0.11)$ for BJ (PS) for non-superfluid models. The resulting modulated $X$ ray flux is $2.2(1.3) \times 10^{-4} / \mathrm{cm}^{2} \mathrm{sec}$ for BJ (PS), or $0.45(0.25)$ of the present limit on modulated $X$ rays from the Vela pulsar. 
TABLE I

Neutron Star Cooling: Calculated Core and Surface Temperatures

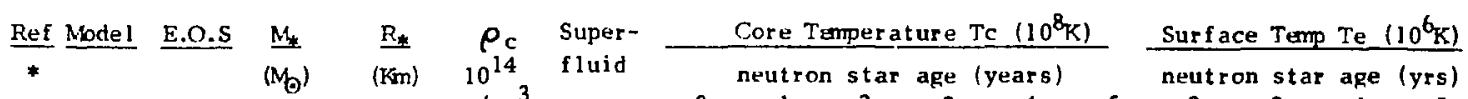

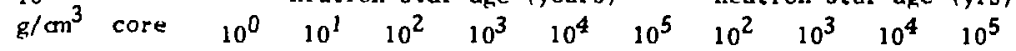

\begin{tabular}{|c|c|c|c|c|c|c|c|c|c|c|c|c|c|c|c|c|}
\hline 23 & V II & medium & 1.07 & 12.3 & 7.4 & yes & 20 & 14 & 9 & 5.0 & 1.6 & 0.1 & 5 & 3 & 1.25 & 0.17 \\
\hline 23 & V II & mediun & 1.07 & 12.3 & 7.3 & no & 16 & 14 & 8 & 5.6 & 4 & 2.5 & 4.4 & 3 & 2.3 & 1.6 \\
\hline 24 & $A^{* *}$ & sof $t$ & 1.32 & 8 & 35 & yes & 28 & 16 & 10 & 8 & 3 & 0.22 & B & 6 & 3 & 0.25 \\
\hline 24 & $A^{* *}$ & sof $t$ & 1.32 & 8 & 35 & no & 10 & 8 & 5.2 & 3.5 & 2.7 & - & 4.7 & 3.5 & 2.7 & 0.7 \\
\hline 25 & PS & stiff & 1.3 & 16.1 & 4 & yes & 16 & 13 & 9 & 5.2 & 3 & - & - & - & - & - \\
\hline 25 & PS & stiff & 1.3 & 16.1 & 4 & no & 10 & 7.4 & 5.1 & 3.5 & 2.2 & - & - & - & - & - \\
\hline 26 & 1 & ***** & 0.253 & 13 & 4.1 & yes & 19 & 13.5 & 7.2 & 3.8 & 2.1 & - & 2.7 & 1.8 & 1.2 & - \\
\hline 26 & IIA & $* * *$ & 0.822 & 10.7 & 9.1 & yes & 23 & 17 & 8.7 & 3.8 & 1.9 & 0.5 & 4.4 & 2.5 & 1.6 & 0.7 \\
\hline 26 & IIB & $* * *$ & 0.822 & 10.7 & 9.1 & no & 11 & 7.2 & 5 & 3.4 & 2.2 & - & 3 & 2.3 & 1.7 & - \\
\hline 26 & III & $\# * *$ & 1.54 & 9.5 & 18.9 & yes & 10 & 8 & 5.6 & 3.7 & 1.9 & - & 3.5 & 2.6 & 1.7 & - \\
\hline
\end{tabular}


Table II

Calculated Hard X Ray Luminosity of the Vela Pulsar

from Axion Conversion in Various Models*

$\begin{array}{lllllllll}\text { Example } & 1 & 2 & 3 & 4 & 5 & 6 & 7 & 8 \\ M_{a}\left(10^{-3} \mathrm{eV}\right) & 2 & 2 & 2 & 2 & 0.6 & 0.6 & 1 & 1\end{array}$

Core nonsuperfl. superfluid nonsuperfl. superfluid

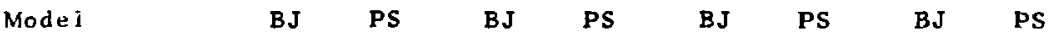

$\begin{array}{lllllllll}R_{*}(\mathrm{Km}) & 11 & 16 & 11 & 16 & 11 & 16 & 11 & 16\end{array}$

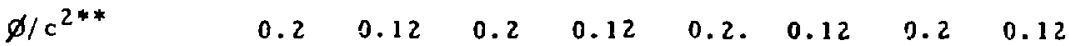

$\begin{array}{llllllllll}B_{0}\left(10^{12} \mathrm{~g}\right) & 2.8 & 1.33 & 2.8 & 1.33 & 2.8 & 1.33 & 2.8 & 1.33\end{array}$

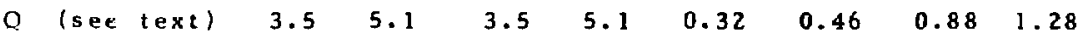

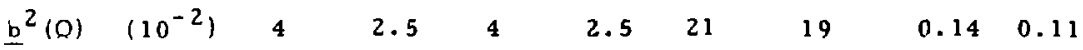

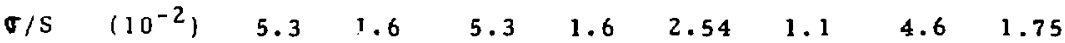

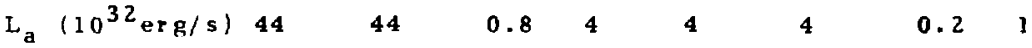

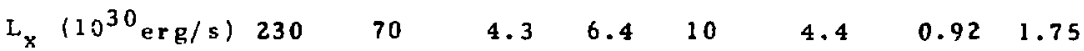

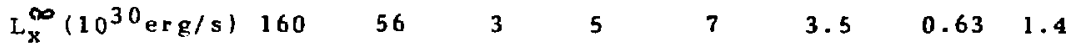

$\begin{array}{llllllll}\begin{array}{l}\text { Fraction of } \\ \text { present limit }\end{array} \quad \frac{1}{4.4} & \frac{1}{12.5} & \frac{1}{230} & \frac{1}{140} & \frac{1}{100} & \frac{1}{200} & \frac{1}{1100} & \frac{1}{500}\end{array}$

* for Core $\mathrm{T}_{\mathrm{C}}=2 \times 10^{8} \mathrm{~K}$

${ }^{* *} L=e^{-2 \phi / c^{2}} L_{x}$ from Ref 14 . 


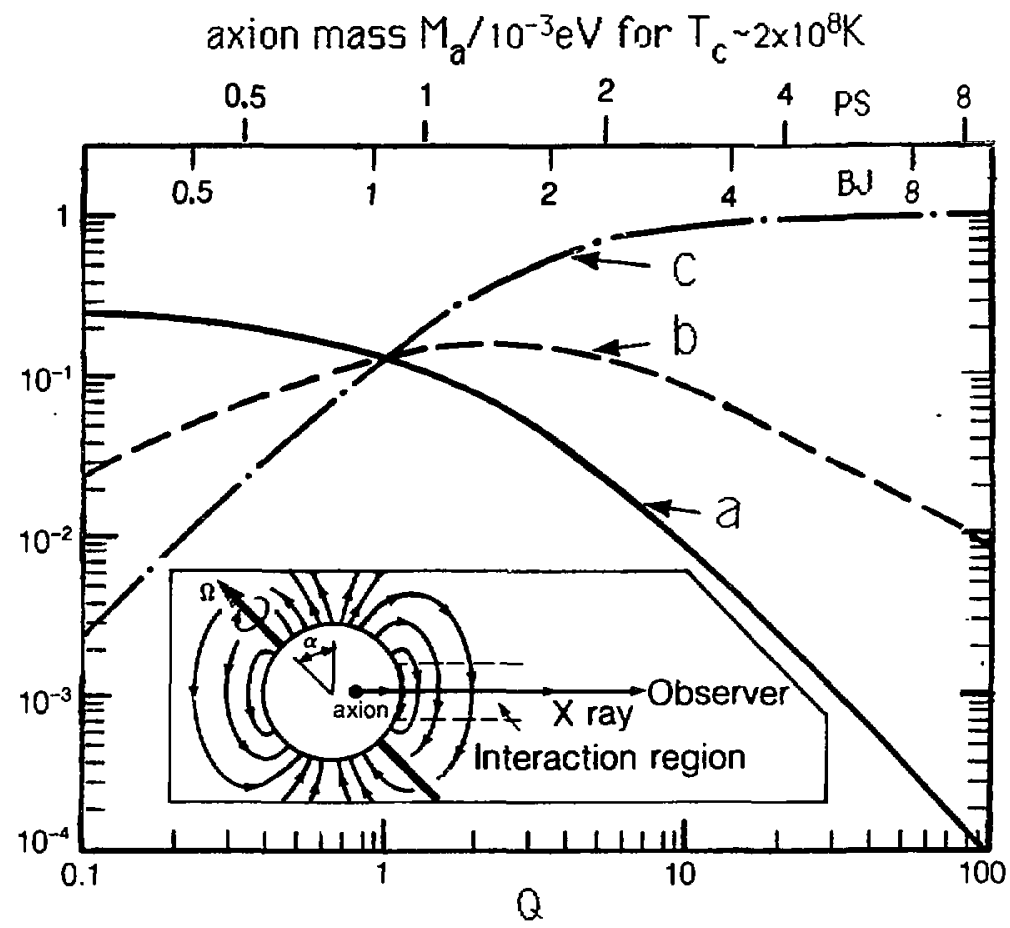

Figure 1. Axion to $\mathrm{X}$ ray Conversion at a neutron star: Dependence on nomalized wave number $O=R_{*} \cdot q=R_{*} \cdot M_{a}{ }^{2} c^{3} / 2 \pitchfork E_{a}$. Corresponding axion mass $M_{a}$ for models $B J$ and $P S$ at $T_{c} \sim 2 \times 10^{8} K$ is indicated (see text).

a. The square of the Fourier transfom of the nomalized magnetic field $\underline{b}^{2}(O)$

b. The axion axion to $X$ ray conversion cross section $\sigma \propto c M_{a}^{2} \underline{b}^{2}(O)$ $\propto O \underline{b}^{2}(O)$, which is plotted.

c. The $x$ ray luminosity fram axion conversion $L_{x}=\eta L_{a} \propto \sigma M_{a}{ }^{2} \propto O^{2} \underline{b}^{2}(O)$, which is plotted. (when $\sigma / S<0.5$ so that $\eta \sim \sigma / S$ ) 
This repon was done with support from the Department of Energy. Any conchusions or opinion expresed in this repon represent solety those of the author(t) and not necesturily those of The Regents of the University of California, the Lewrence Berkeky Leboratory or the Department of Encrey.

Reference to a company or product rame does not imply approval or rocommendation of the product by the University of Celifornis or the U.S. Department of Energy to the cxclusion of others that may be suivable. 